\title{
Pressure dependence of the spin dynamics around a quantum critical point : An inelastic neutron scattering study of $\mathrm{Ce}_{0.87} \mathrm{La}_{0.13} \mathbf{R u}_{2} \mathrm{Si}_{2}$.
}

\author{
S. Raymond $\dagger$, L.P. Regnault $\dagger, J$. Flouquet $\dagger$, A. Wildestand P. \\ Lejay§ \\ $\dagger$ CEA-Grenoble, DRFMC / SPSMS / MDN, 38054 Grenoble Cedex, France \\ $\ddagger$ Institut Laue Langevin, 38042 Grenoble Cedex, France \\ $\S$ CRTBT-CNRS, 38042 Grenoble Cedex, France
}

\begin{abstract}
.
Inelastic neutron scattering experiments performed on a single crystal of the antiferromagnetic compound $\mathrm{Ce}_{0.87} \mathrm{La}_{0.13} \mathrm{Ru}_{2} \mathrm{Si}_{2}$ under applied pressures of up to 5 kbar are reported. A quantum critical point is reached at around 2.6 kbar where longrange magnetic order disappears. The variation of the characteristic energy scales with respect to temperature and pressure is followed and found to saturate in the ordered phase.
\end{abstract}




\section{Introduction}

Much experimental and theoretical work has been devoted to the study of quantum phase transitions in recent years [1, 2, 3, 4]. Such a transition from an ordered to a disordered state occurs at zero temperature as a function of a control parameter $r$ (pressure $P$, magnetic field $H$, impurity concentration $x$ ). Heavy fermion (HF) compounds provide an opportunity to study such phenomena since a variety of ground states, from weak antiferromagnetic to Pauli paramagnetic states, can be realized by tuning $r$. HF physics has been long understood from the point of view of the socalled Doniach phase diagram describing the competition between the formation of a non-magnetic Kondo singlet and the realization of an ordered state via the RKKY (Ruderman-Kittel-Kasuya-Yosida) interactions [5]. Renewed interest in this field has came about from precise studies of the quantum critical point (QCP) in itinerant magnets and the marginal behavior observed near the QCP, in particular the so-called non-Fermi liquid (NFL) behavior [1, 4.

Only a few studies have been performed on single crystals using inelastic neutron scattering (INS). The study of a single crystalline sample on a three-axis spectrometer (TAS) allows the measurement of the full $(\mathbf{Q}, \omega)$ dependence of the imaginary part of the dynamical spin susceptibility $\chi^{\prime \prime}(\mathbf{Q}, \omega)$. At present, only two systems were extensively studied near the QCP : $\mathrm{CeCu}_{6}$ doped with $\mathrm{Au}$ on the $\mathrm{Cu}$ site [6] and $\mathrm{CeRu}_{2} \mathrm{Si}_{2}$ doped with La on the Ce site[7]. For the latter systems experiments with Rh substitution on the $\mathrm{Ru}$ site were also performed [8]. In these works, the magnetic excitation spectrum of two samples were studied, one in the paramagnetic phase $\left(x<x_{c}\right)$ and one located near the instability point $\left(x=x_{c}\right)$. While these two studies bear similar experimental results : reduction of the energy scale near the QCP and increase of the correlation length, emphasise was put on different points. For $\mathrm{CeCu}_{6-x} \mathrm{Au}_{x}$, the anisotropy of the magnetic response [9] was put forward to explain the NFL behavior observed in bulk measurements and $\omega / T$ scaling was found in the dynamical spin susceptibility [10]. In the latter $\mathrm{Ce}_{1-x} \mathrm{La}_{x} \mathrm{Ru}_{2} \mathrm{Si}_{2}$ system, the accent was put on the Self Consistent Renormalized Spin Fluctuation (SF) theory of Moriya [3] which allows to link the magnetic excitation spectrum to the bulk measurements and their evolution towards the QCP [11, [1].

Here we propose a different experimental approach starting from an antiferromagnetic compound and studying the evolution of the magnetic excitation spectrum with pressure up to $5 \mathrm{kbar}$. This spans the phase diagram through the QCP at finite temperature. The main advantage is that the same crystal is used throughout, avoiding the problem of disorder which is difficult to handle when results obtained on crystals with different concentration $x$ are compared. The disadvantage is that the temperature range is limited by the experimental setup (pressure cell) both on the lower and higher temperature sides. 


\section{Experimental details}

$\mathrm{CeRu}_{2} \mathrm{Si}_{2}$ crystallizes in the body centred tetragonal $I 4 / \mathrm{mmm}$ space group with the lattice parameters $a=b=4.197 \AA$ and $c=9.797 \AA$. The dependence of these parameters on La concentration is roughly linear and of the order of $5 \cdot 10^{-4} \stackrel{\circ}{A}$ at. \% La. The crystal studied here with $x=0.13$, grown by the Czochralsky method, has a volume of $250 \mathrm{~mm}^{3}$ 13 .

Experiments were carried out on the cold TAS IN14 at the ILL high flux reactor, Grenoble. A first set of measurements was performed in a standard orange cryostat at ambient pressure and a second set was carried out in an helium transmitting medium pressure cell made of $\mathrm{Al}$ alloy in a large He flow orange cryostat. The experimental conditions were the same for both experiments using the constant final energy mode with $\mathrm{k}_{F}=1.97 \AA^{-1}$. The collimations were open-40'-60'-60' and a graphite filter was used in order to reduce higher order contamination. With this setup the width of the incoherent peak (Full Width at Half Maximum (FWHM) of a gaussian profile) was $0.35 \mathrm{meV}$. These conditions were chosen to minimize the background (flat analyzer and collimations) which appears to be crucial for the experiment in the pressure cell. A window of Cadmium (neutron absorber) was put around the cryostat in order to cut the background of the pressure cell. Measurements were performed above $2.6 \mathrm{~K}$ to avoid the presence of superfluid He from the flow around the pressure cell.

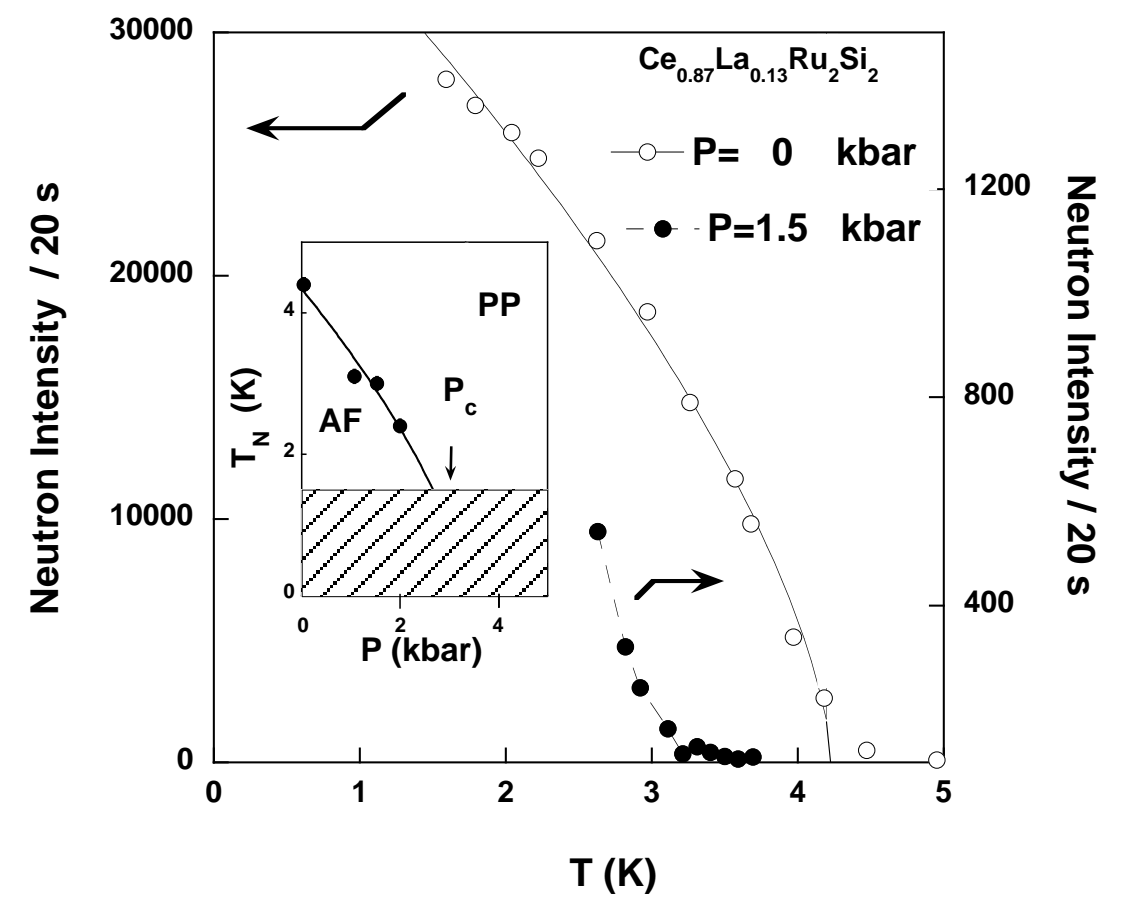

Figure 1. Neutron intensity versus temperature measured at $\mathbf{Q}=(0.69,1,0)$ at $P=0$ and $1.5 \mathrm{kbar}$. The lines are guides for the eyes. The inset shows the pressure variation of the Néel temperature. The line corresponds to the power law fit explained in the text. The hatched area corresponds to the temperature range not coverd by this experimental setup. 


\section{Determination of the critical pressure}

Magnetic ordering occurs in $\mathrm{Ce}_{1-x} \mathrm{La}_{x} \mathrm{Ru}_{2} \mathrm{Si}_{2}$ for $x \geq 0.08\left(x_{c}=0.075\right)$. The ordering takes the form of a sine-wave modulated structure with the incommensurate wave vector $\mathbf{k}=(0.31,0,0)$ and the magnetic moments along the $c$ axis [14]. For the sample studied here $(x=0.13)$, the Néel temperature is $T_{N}=4.4 \mathrm{~K}$ with a magnetic moment of $1.05 \mu_{B}$ at $1.5 \mathrm{~K}$ and at ambiant pressure. The $(P, T)$ phase diagram of this sample was drawn by studying the evolution of the magnetic Bragg peak $\mathbf{Q}=(0.69,1,0)$ as a function of pressure $(\mathbf{Q}=\tau$ - $\mathbf{k}$ where $\tau=(1,1,0)$ is a reciprocal lattice translation.). The measured neutron intensity versus temperature at ambient pressure without the cell and at $1.5 \mathrm{kbar}$ in the pressure cell is shown in Fig.1. The pressure variation of the Néel temperature is shown in the inset of Fig.1. The data below $2.6 \mathrm{~K}$ came from a preliminary experiment performed with similar experimental conditions at Laboratoire Léon brillouin, Saclay, France. The agreement between several sets of data give the experimental precision between two different series of experiments. The overall data show the reliability of the pressure cell. Above $2.8 \mathrm{kbar}$, no sign of magnetic ordering is found down to 1.8

$\mathrm{K}$. If the pressure variation of the Néel temperature is fitted according to the relation expected for a 3 dimensionnal antiferromagnet near the QCP :

$$
T_{N} \propto\left(P-P_{c}\right)^{2 / 3},
$$

the critical pressure is $P_{c}=3.4 \mathrm{kbar}$. It is also predicted that the $T=0$ staggered magnetic moment $m_{k}$ will follow the relation :

$$
m_{k} \propto\left(P-P_{c}\right)^{1 / 2}
$$

From our measurements, it is clear that $m_{k}$ does not follow a simple law like (2). This quantity rapidly drops at low pressure and then has the tendency to saturate. Such a behavior was preciselly studied for alloys of the family $\mathrm{Ce}\left(\mathrm{Ru}_{1-x} \mathrm{Rh}_{x}\right)_{2} \mathrm{Si}_{2}$ [15, [16] (This is not the purpose of the present work which concentrates on the dynamics). It is thus difficult to extract $P_{c}$ from the pressure variation of $m_{k}$, an upper estimate would be $2.2 \mathrm{kbar}$. Another estimate of $P_{c}$ can be given from the bulk measurements performed on several concentrations or under pressure in this family of compounds. Resitivity measurements performed for $x=0.2$ under pressure show that $2.4 \%$ of dilution corresponds to $1 \mathrm{kbar}$ [17]. For the compound studied here with $x=0.13$, this will give an expected $P_{c}$ of $2.1 \mathrm{kbar}$. In the following, we will take the mean value of these three different estimates : $P_{c}=2.6 \pm 0.5$ kbar.

\section{Magnetic excitation spectrum at $P=0$}

The magnetic excitation spectrum is shown in Fig.2 for several $\mathbf{Q}$ vectors $\left(\mathbf{Q}=\left(Q_{H}, 1,0\right)\right.$ where $Q_{H}$ is expressed in reciprocal lattice units (r.l.u.)) at $2.6 \mathrm{~K}$. The corresponding wave-vector response is shown in the inset of Fig.2 for two energy transfers of 0.8 and $1.5 \mathrm{meV}$. The signal is peaked at $Q_{H}=0.69$ for both energies and the lineshape broadens when energy increases. The response in energy is quasielastic as shown in Fig.2 for 


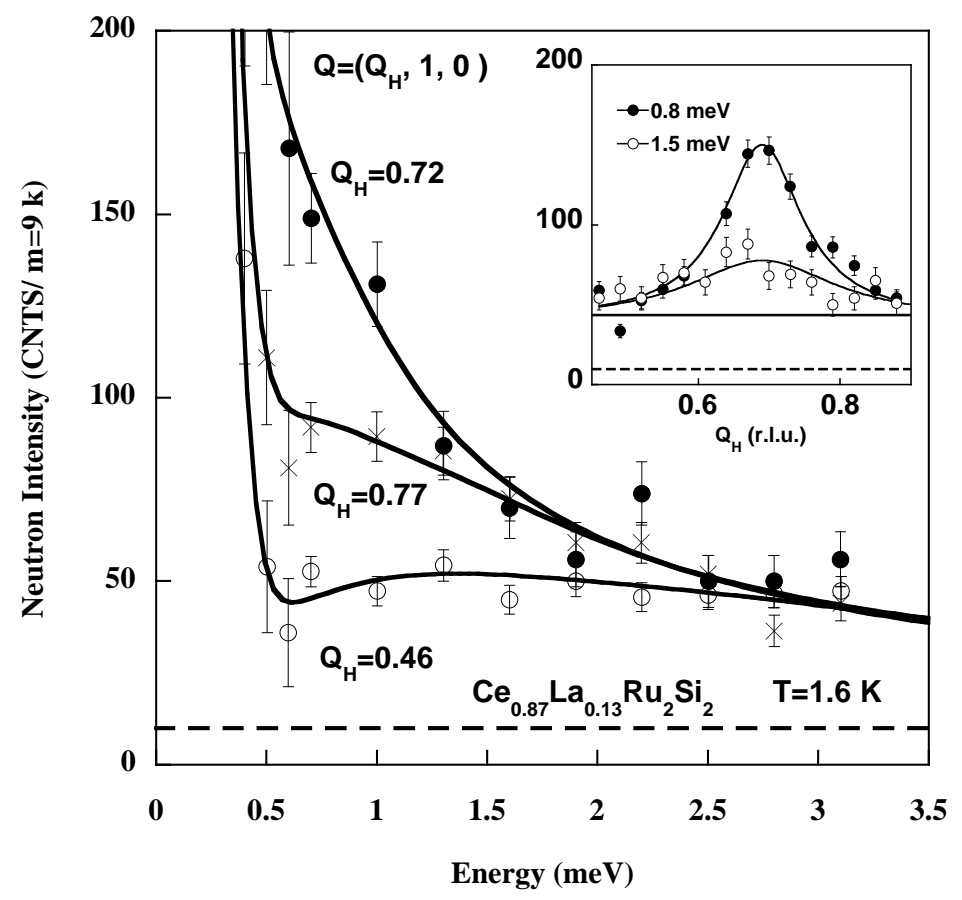

Figure 2. Constant $\mathbf{Q}$ scans realized at $2.6 \mathrm{~K}$ and $P=0 \mathrm{kbar}$ around the wavevector of instability k. Lines corresponds to the fit explained in the text. The inset shows two constant energy scans performed at 0.8 and $1.5 \mathrm{meV}$. The dashed line indicates the background of the spectrometer. The solid straight line in the inset corresponds to the single site contribution.

$Q_{H}=0.72$. This wave-vector is chosen to be $\mathbf{k}+(0.03,0,0)$ where $\mathbf{k}$ is the wave-vector of instability in order to avoid strong Bragg contamination. This offset has no consequences on the determined physical quantities (energy widths) since the signal we measured does not vary rapidly around $\mathbf{k}$ in the energy range probed with our instrumental resolution. Despite the fact that the compound is ordered, the spectrum is characteristic of SF and no well defined (single mode) excitations are observed. The background of the spectrometer (determined for negative energy transfer at low temperature) does not correspond to the background of the constant $\omega$ scan suggesting the existence of a $\mathrm{Q}$ independent (or single-site) contribution as in other compounds of this familly [11, 19, 20. Consequently, we used the same procedure as in the previous works on $x=0$ 20] and $x_{c}=0.075$ [11] for a quantitative analysis of the data. This procedure accounts for the background in a consistent way for both constant $\mathbf{Q}$ and constant $\omega$ scans. In the first works on $\mathrm{CeRu}_{2} \mathrm{Si}_{2}$, the single-site contribution was not taken into account [18 since only high magnetic field studies unambiguously underline the existence of this contribution [19, 20]. By applying a magnetic field, the correlated part of the signal vanishes and only the $\mathbf{Q}$ independent contribution persists at the same level of intensity that was determined at $H=0$. In a similar fashion to the applied external magnetic field case, we will also show that this decomposition of the magnetic scattering into singlesite and correlated signal is very convenient for understanding experiments carried out 
Spin dynamics around a quantum critical point in $C e_{0.87} \mathrm{La}_{0.13} R u_{2} \mathrm{Si}_{2}$

Table 1. Characteristic energies (in meV) measured at $2.6 \mathrm{~K}$ on several compounds $\mathrm{Ce}_{1-x} \mathrm{La}_{x} \mathrm{Ru}_{2} \mathrm{Si}_{2}$ for $\mathbf{Q}=\mathbf{k}$ with similar experimental conditions (cold TAS).

\begin{tabular}{llll}
\hline $\mathrm{x}$ & $\Gamma_{I S}$ & $\omega_{0}$ & $\Gamma_{S S}$ \\
\hline 0 & $0.77(5)$ & $0.5(1)$ & $2.0(1)$ \\
0.075 & $0.17(2)$ & $0.2(1)$ & $1.4(1)$ \\
0.13 & $0.15(3)$ & $0.10(5)$ & $1.4(2)$ \\
\hline
\end{tabular}

under pressure. In this approach, the neutron intensity is written :

$$
I(\mathbf{Q}, \omega)=I_{B G}+\left(1+n_{B}(\omega)\right)\left(\chi_{S S}^{\prime \prime}(\omega)+\chi_{I S}^{\prime \prime}(\mathbf{Q}, \omega)\right)
$$

where $I_{B G}$ is the background intensity, $n_{B}(\omega)=1 /\left(e^{\omega / T}-1\right)$ is the Bose factor and $\chi_{S S}^{\prime \prime}$ and $\chi_{I S}^{\prime \prime}$ are respectively the imaginary part of the single site and intersite magnetic dynamical susceptibility. The single-site contribution is assumed to be Lorentzian, reflecting local $4 f$ spin relaxation :

$$
\chi_{S S}^{\prime \prime}(\omega)=\chi_{S S}^{\prime} \frac{\omega \Gamma_{S S}}{\omega^{2}+\Gamma_{S S}^{2}}
$$

where $\chi_{S S}^{\prime}$ is the local susceptibility and $\Gamma_{S S}$ is the local fluctuation rate. It is related to the Kondo temperature $T_{K}\left(\Gamma_{S S} \approx k_{B} T_{K}\right.$ [21]). For the correlated signal, we use the following formula were $\mathbf{q}=\mathbf{Q}-\tau-\mathbf{k}$ :

$$
\chi_{I S}^{\prime \prime}(\mathbf{q}, \omega)=\frac{\chi_{I S}^{\prime}(\mathbf{q})}{2} \omega\left(\frac{\Gamma_{I S}(\mathbf{q})}{\left(\omega-\omega_{0}(\mathbf{q})\right)^{2}+\Gamma_{I S}^{2}(\mathbf{q})}+\frac{\Gamma_{I S}(\mathbf{q})}{\left(\omega+\omega_{0}(\mathbf{q})\right)^{2}+\Gamma_{I S}^{2}(\mathbf{q})}\right)(5)
$$

where $\chi_{I S}^{\prime}(\mathbf{q})$ is the $\mathbf{q}$ dependent part of the suceptibility, $\Gamma_{I S}(\mathbf{q})$ is the intersite fluctuation rate and $\omega_{0}(\mathbf{q})$ is an inelastic energy which clearly better describes the data obtained for compounds of this familly located in the paramagnetic region [20]. In this paper, we will focus on the response in energy at the wave vector $\mathbf{k}$ and will note $\Gamma_{I S}=\Gamma_{I S}(q=0)$. It is also found that $\omega_{0}(\mathbf{q})$ does not depends of $\mathbf{q}$ as was already known from studies of other compositions.

The single site contribution is unambiguously determined at $\mathbf{Q}=(0.46,1,0)$ in a part of the Brillouin zone where the signal is flat in $\mathbf{q}$ (see inset of Fig.2) and thus $\chi_{I S}^{\prime}=0$. The fit to the data is shown in Fig.2. Since the neutron intensity is not normalized, we are only interested in the energy width, $\Gamma_{S S}=1.4(2) \mathrm{meV}$. A fit at the vector $\mathbf{k}$ is also shown in Fig.2. We found for the offset $q_{H}=0.03\left(\mathbf{q}=\left(q_{H}, q_{K}, q_{L}\right)\right)$ at $2.6 \mathrm{~K}$ in the ordered phase, $\Gamma_{I S}=0.15$ (3) $\mathrm{meV}$ and $\omega_{0}=0.10$ (5) meV. Contrarily to the pure compound $\mathrm{CeRu}_{2} \mathrm{Si}_{2}$, the determination of $\omega_{0}$ lies in the limits of the fit since it is smaller than the resolution. It is worth noting that the determined value of $\Gamma_{I S}$ does not change much if $\omega_{0}$ is fixed to zero. For comparison, the values obtained at $2.6 \mathrm{~K}$ for the compounds corresponding to $x=0$ and $x_{c}=0.075$ are shown in Table 1 . The $q_{H}$ dependence was described by expanding $\Gamma_{I S}\left(q_{H}\right)=\Gamma_{I S}\left(1+\left(q_{H} / \kappa\right)^{2}\right)$ in (5) and assuming that $\chi_{I S}\left(q_{H}\right) \Gamma_{I S}\left(q_{H}\right)=$ constant 22]. The fit to the data obtained at 0.8 and $1.5 \mathrm{meV}$ are shown in the inset of Fig.2 with a value of $\kappa=0.05$ r.l.u. This corresponds to a 
correlation length $\xi \approx 13 \AA$ that is around 3 lattice units. This is the correlation length of the remaining longitudinal fluctuations after magnetic order is established in this system. This must not to be confused with the correlation length introduced in phase transition theory which diverges at $T_{N}$ or at $P_{c}$. This latter quantity needs to be measured in a double-axis configuration in order to have an energy integrated signal.

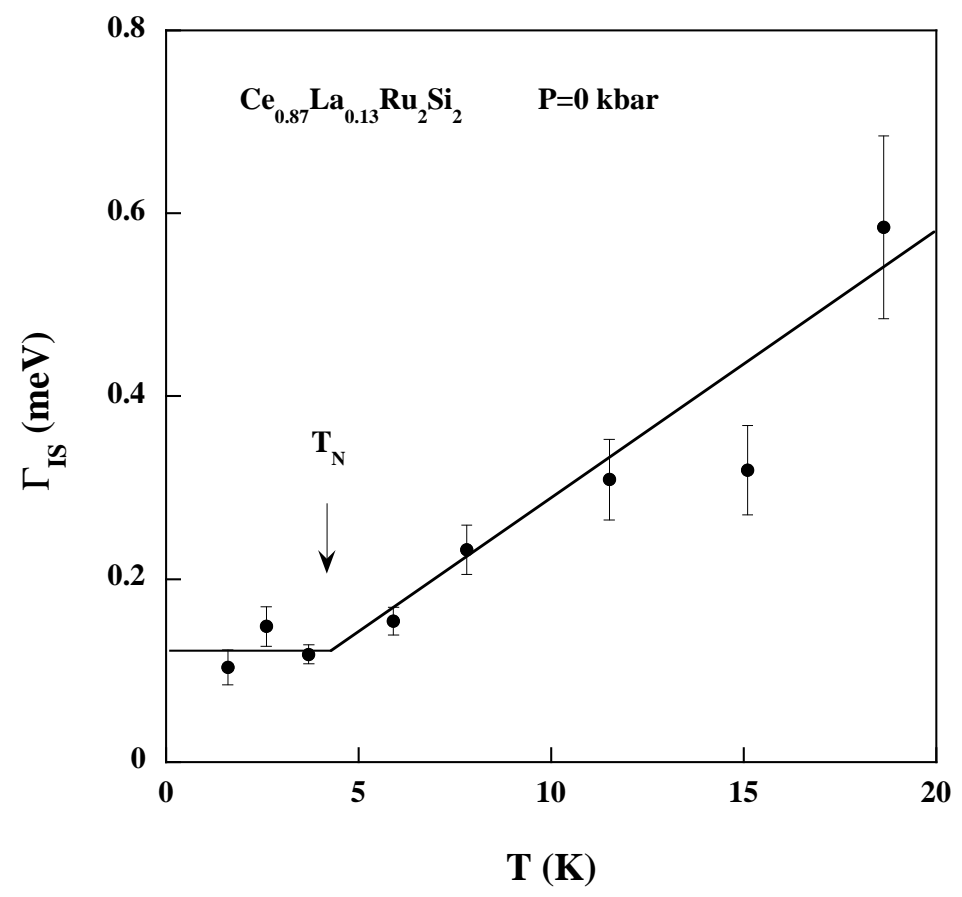

Figure 3. Temperature dependence of the fluctuation rate $\Gamma_{I S}$ measured at $P=0$ kbar. The line is a guide for the eyes.

The analysis was repeated for several temperatures. On increasing $T$, the magnetic excitation spectrum broadens continuously. Of interest is the temperature variation of $\Gamma_{I S}$. This quantity is shown in Fig. 3. The temperature variation is almost linear above $T_{N}$, slowing down and eventualy saturating below $T_{N}$. In contrast, the quantity $\Gamma_{S S}$ is almost temperature independent in the studied range of 1.5-20 K. At higher temperature, for $\Gamma_{S S}>k_{B} T$, it is expected that $\Gamma_{S S}$ will acquire some temperature dependence [21].

\section{Evolution of the magnetic excitation spectrum with pressure}

Similarly to the temperature dependence studied at zero pressure, we also studied the evolution of the spin dynamics with pressure at constant temperature of 2.6 K. Fig. 团 shows the neutron intensity measured versus pressure for two energy transfers of 1 and $6 \mathrm{meV}$. The spectrometer background measured for an energy transfer of $-1 \mathrm{meV}$ at 2.6 $\mathrm{K}$ is subtracted. These data show that the low energy response decreases when pressure increases while the high energy part is not sensitive to pressure. A crossover between low energy and high energy dynamics therefore needs to be defined. In the framework 


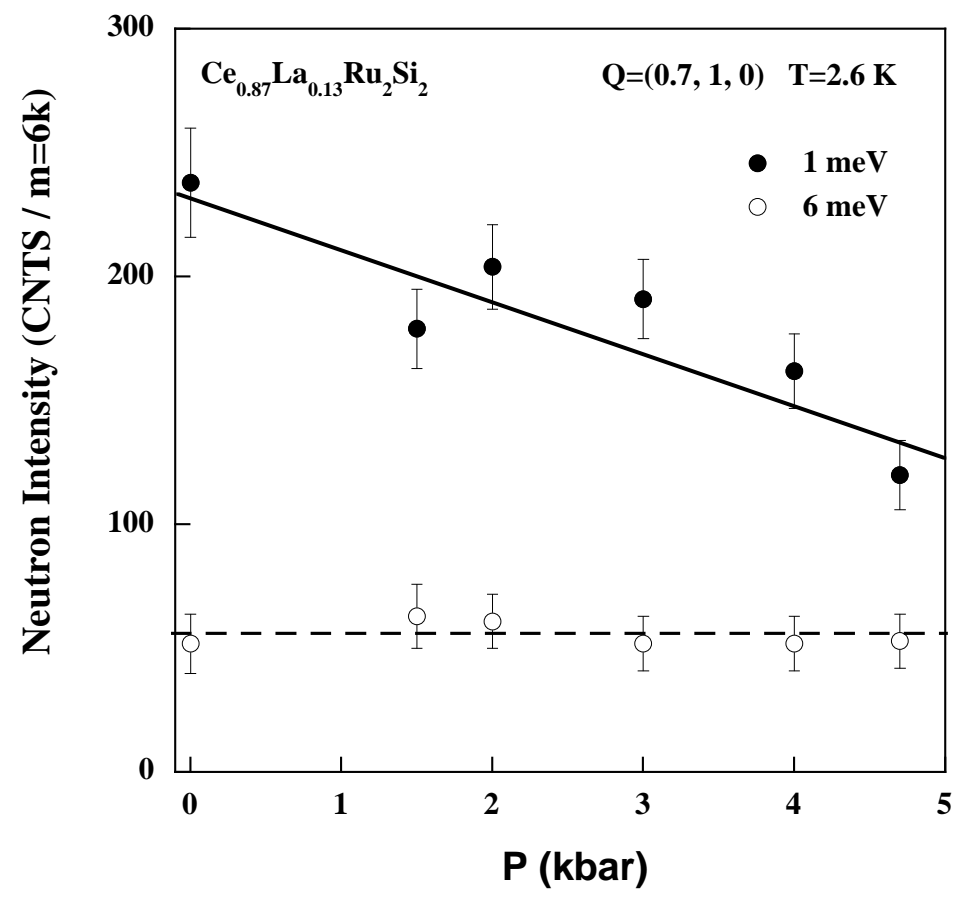

Figure 4. Pressure dependence of the inelastic magnetic scattering measured at $\mathbf{Q}=(0.7,1,0)$ for $1 \mathrm{meV}$ (full circle) and $6 \mathrm{meV}$ (open circle) energy transfer at 2.6 K. Spectrometer background is subtracted. Lines are guides for the eyes.

of quantum phase transition and for a gapless system [4], this crossover energy $\Delta$ is the relevant quantity to study the QCP (i.e. $\Delta \rightarrow 0$ at the QCP). The analysis of the dynamical spin susceptibility of this system in a single-site and inter-site contributions provides us with a natural way of defining such a crossover. Indeed, the high energy part corresponds to a single-site only contribution. In the following, we will simply take $\Delta \approx \Gamma_{I S}$. In this respect both pressure and magnetic field suppress the inter-site contribution in a similar ways (See Fig.8 of Ref [20] in comparison with Fig.(4).

Fig.5 shows the evolution of the inter-site signal measured at $Q=(0.7,1,0)$ after subtraction of the data from the single-site signal measured at each pressure at $\mathbf{Q}=(0.46,1,0)$. At zero pressure (upper frame), a Bragg contribution appears at negative energy transfer due to the choice of the focusing conditions (offset $q_{H}=0.01$ ). The inelastic signal broadens with increasing pressure and at $4.7 \mathrm{kbar}$ it is very similar to the one of the pure compound $\mathrm{CeRu}_{2} \mathrm{Si}_{2}$ (see Fig.9 in Ref. [20]).

The pressure variation of $\Gamma_{I S}$ is shown in Fig.6a. The data obtained at the same temperature for the alloy corresponding to the critical concentration, $x_{c}=0.075$, and to the pure compound, $x=0$, are shown in the same plot with the concentration-pressure conversion explained in the first section. There is an acceptable agreement with these former measurements and our present measurements under pressure. The data presented in this way exhibit some noise due to the fact that they were taken from different experiments with different setups. There is thus no correlations between the error bars for the points obtained under pressure and for the ones obtained on the alloys. All the 
Spin dynamics around a quantum critical point in $C e_{0.87} L a_{0.13} R u_{2} S i_{2}$

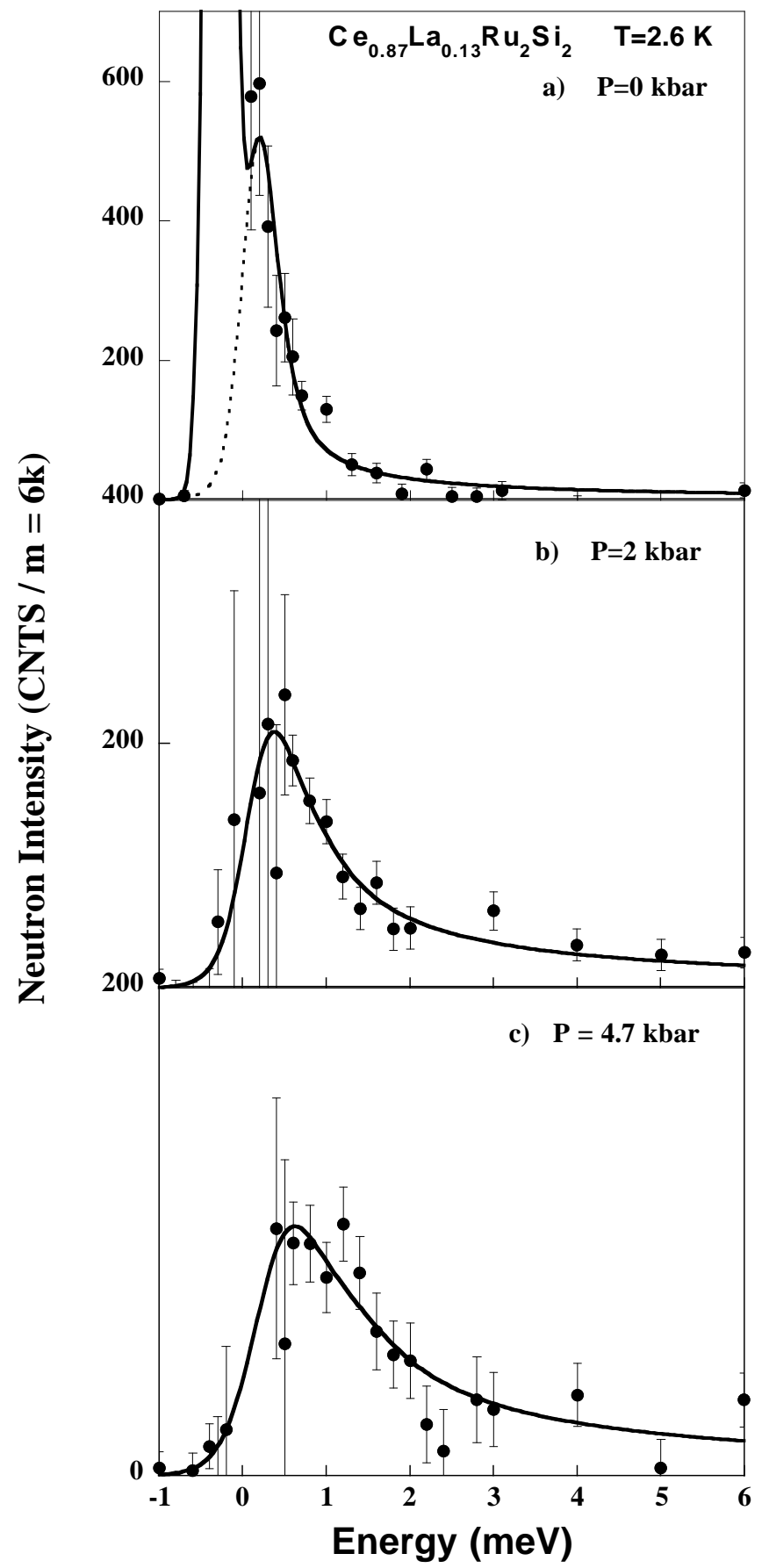

Figure 5. Evolution with pressure of the intersite contribution of the dynamical spin susceptibility measured at $2.6 \mathrm{~K}$ for 0,2 and $4.7 \mathrm{kbar}$. The experimental data are obtaied by point by point subtraction of $\mathbf{Q}=(0.7,1,0)-\mathbf{Q}=(0.46,1,0)$

more, the data taken under pressure lead also to higher error bars since the background is higher. Nevertheless, this correct agreement stresses the validity of the analogy between concentration and pressure in the limit of small disorder.

Despite the limited statistics of the data, it seems that the energy width saturates below the critical pressure $P_{c}$ as it does below $T_{N}$ at $P=0$. Indeed the two sets of data 
$\Gamma_{I S}(P)$ and $\Gamma_{I S}(T)$ are strikingly similar. The data clearly show that the increase of $\Gamma_{I S}$ is much higher in the non-magnetic phase above $3 \mathrm{kbar}$. To reproduce this behavior, we made a global fit of these data to the phenomenological expression :

$$
\Gamma_{I S}(P)=\Gamma_{I S}(0)+\alpha P \exp \left(-P^{*} / P\right)
$$

with $\Gamma_{I S}(0)=0.19(2) \mathrm{meV}, \alpha=0.4(2) \mathrm{meVkbar}^{-1}$ and $P^{*}=7$ (2) kbar. This expression reproduces the saturation of $\Gamma_{I S}$ at low pressure and is linear in $P$ for $P \gg P^{*}$. The pressure variation of $\Gamma_{S S}$ is shown in Fig.6b. This quantity increases only slightly in the pressure range studied. This confirms the idea that high energy dynamics do not change much with pressure. The data are phenomenologicaly described by the smooth variation $\Gamma_{S S}=\Gamma_{S S}(0)+\eta P^{2}$ with $\Gamma_{S S}(0)=1.3(1) \mathrm{meV}$ and $\eta=0.025$ (5) $\mathrm{meVkbar}^{-2}$. Finally, the pressure variation of $\omega_{0}$ is shown in the inset of Fig. G $\mathrm{b}$. It is very similar to the one of $\Gamma_{I S}$. The relation $\omega_{0} \propto 0.6 \Gamma_{I S}$ approximately holds. Beyond these phenomenological descriptions, an order of magnitude can be extracted for the pressure varition of the different characteristic energies $\epsilon\left(\epsilon=\Gamma_{I S}, \Gamma_{S S}\right.$ or $\left.\omega_{0}\right)$. When taken the pressure range studied $\Delta P$ as a whole, all these quantities increase with a quite similar rate of $\Delta \epsilon / \Delta P \approx$ $1 \mathrm{Kkbar}^{-1}$.

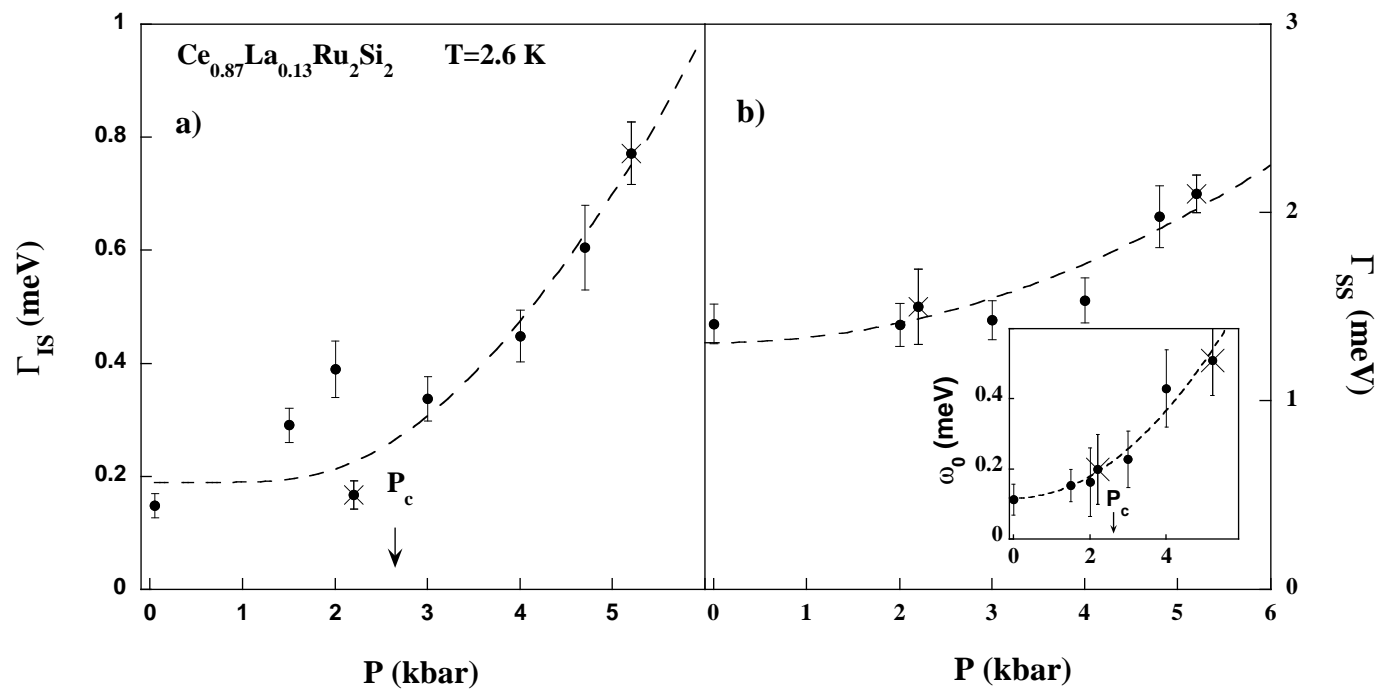

Figure 6. a) Pressure dependence of the fluctuation rate $\Gamma_{I S}$ measured at $2.6 \mathrm{~K} . \mathrm{b}$ ) Pressure dependence of the local fluctuation rate $\Gamma_{S S}$ measured at $2.6 \mathrm{~K}$. The inset shows the pressure dependence of $\omega_{0}$. For each panel, the crossed circles correspond to data obtained on the alloys $x=0$ and $x_{c}=0.075$ with the pressure-concentration conversion explained in the text. Lines correspond to fit explained in the text.

\section{Discussion}

\subsection{Nature of the excitations}

We first discuss the nature of the excitations in the ordered phase. Due to the strong Ising nature of the system [23], the observation of spin waves by INS is precluded. The 
observed excitations correspond to fluctuations of the order parameter (longitudinal fluctuations). This was previously established by INS on $\mathrm{CeRu}_{2} \mathrm{Si}_{2}$ [24]. The fact that the excitations are not well-defined dispersive modes is understood from the magnetic structure of the system. For an incommensurate structure, $\mathbf{q}$ is not a good quantum number due to the lack of translational invariance. INS measurements probe the dynamical susceptibility at $(\mathbf{q}, \omega)$ and this corresponds here to the coupling of several eigen-modes giving a broad signal [25]. This is not the case for real antiferromagnetic structure where longitudinal well-defined dispersive modes can be observed in compounds with weak magnetic moments. Such modes were measured by INS in $\mathrm{URu}_{2} \mathrm{Si}_{2}$ 26].

Our data show similar results when the ordered phase is reached either by varying temperature or pressure. The fluctuation rate seems to reach a constant and finite value in the ordered phase. This behavior can be partly understood from the point of view of the magnetic sum rule which states that the total magnetic scattering integrated over $\mathbf{q}$ and $\omega$ is proportional to the square of the magnetic moment of the ion (in fact $S(S+1)$ in the quantum mechanical treatment of a spin $S$ ). Since the ordered moment at $T \approx$ $0, P \approx 0\left(m_{k}=1.05 \mu_{B}\right)$ does not reach the saturated value $\left(m_{\text {sat }}\right)$ determined by the crystal field ground state $\left(\approx 1.7 \mu_{B}\right)$, and since spin-waves are not present, the presence of longitudinal fluctuations is necessary in order to satisfy the magnetic sum rule. In particular, we do not observe any complete softening either of $\Gamma_{I S}(P)$ at $P_{c}$ or of $\Gamma_{I S}(T)$ at $T_{N}$. This can also be partly understood with the same argument. This is also linked to the itinerant nature of the magnetic order, as discussed in the following subsection.

\subsection{Analysis in a spin fluctuation approach}

Our data suggest that the magnetic order has the form of a spin density wave at least down to the lowest measured temperature of $1.5 \mathrm{~K}$. Firstly, we are able to accurately follow the local fluctuation rate, $\Gamma_{S S}$ as a function of pressure, which does not vanish at the QCP but smoothly decreases from the disordered to the ordered state. This quantity reflects the mechanism of local relaxation of $4 f$ moments that is the Kondo effect. This implies that there is no breakdown of the Kondo effect at $P_{c}$ in the $\mathrm{Ce}_{1-x} \mathrm{La}_{x} \mathrm{Ru}_{2} \mathrm{Si}_{2}$ system. Secondly, the magnetic order realized for $x=0.13$ is purely sinusoidal since no higher harmonics were found down to the lowest temperatures [27]. This also points towards a SDW picture since such a pure sinusoidal modulation is typical of itinerant magnetism while squaring of the modulation occurs in localized spin systems. Indeed, in $\mathrm{Ce}_{1-x} \mathrm{La}_{x} \mathrm{Ru}_{2} \mathrm{Si}_{2}$, higher harmonics were found to develop for $x \geq$ 0.2 [28]. Such a crossover from a SDW to a local magnetism is also observed in the similar $\mathrm{Ce}\left(\mathrm{Ru}_{1-x} \mathrm{Rh}_{x}\right)_{2} \mathrm{Si}_{2}$ compounds [29]. The underlying hypothesis of an itinerant magnetism description is that a Fermi surface is well defined throughout the whole phase diagram.

In the past years, neutron scattering data and bulk measurements obtained on the $\mathrm{Ce}_{1-x} \mathrm{La}_{x} \mathrm{Ru}_{2} \mathrm{Si}_{2}$ system were self consistently analyzed in the Moriya's SF theory 
[11, 12]. The finite fluctuation rate measured by neutron scattering around $P_{c}$ must be obviously related to the observation that the resistivity always reaches a $T^{2}$ dependence at the lowest temperature and that concomitently, the specific heat is always linear in $T$. Our new INS data confirm such a picture. The dynamical spin susceptibility, which is a phenomenological starting point of SF theory, was also recently deduced form a microscopic model taking equally into account both the Kondo effect and the RKKY interactions ; the INS cross section derived is very similar to the one used in this paper [30, 31].

\subsection{Comparison with $\mathrm{CeCu} u_{6-x} A u_{x}$}

In the $\mathrm{CeCu}_{6-x} \mathrm{Au}_{x}$ system, the QCP is reached for $x \approx 0.1$. NFL behavior observed either at $P_{c}$ or $x_{c}$ in this system (linear resistivity, logarithmic divergence of the specific heat) was extensively studied by the Karlsruhe group [6]. It is believed that such a behavior implies a new theoretical treatement of the QCP in a strong coupling approach, a local picture where the Fermi liquid description breaks down [32]. The underlying idea is that both $T_{N}$ and $T_{K}$ go to zero at the QCP : the ordered phase is characteristic of local magnetism. Concerning INS, the landmark of such a behavior is the so-called $\omega / T$ scaling of the dynamical spin susceptibility [10]. In the description developped here, this means that $\Gamma_{I S}$ equals $k_{B} T$ (no single-site signal was identified in these studies [9, 10].). This obviously implies that (i) The fluctuation rate totally softens at the QCP团 and (ii) $\omega$ and $T$ are similarly weighted in the spin dynamics. On the contrary, in the SF approach, the fluctuation rate $\Gamma_{I S}$ is written $y_{0}+a^{\prime} T^{3 / 2}\left[\right.$, 31, 34] where $y_{0} \rightarrow 0$ at the QCP and $a^{\prime}$ is constant. Following this argument, $\omega / T^{3 / 2}$ scaling would be expected for a 3 dimensional system near the QCP (in the cas $a^{\prime} \approx 1$ ). This difference with the observed $\omega / T$ scaling in $\mathrm{CeCu}_{5.9} \mathrm{Au}_{0.1}$ has a deeper meaning. In the itinerant magnetism model [2], a system in the vicinity of a QCP is above the critical dimension $\left(d_{c}=4\right.$ above which the Landau theory is valid). This is linked to the increasing importance of the fluctuations in time at $T=0$ near the QCP. Formaly, this is described by the dynamical exponent $z$ ( $z=2$ for an itinerant antiferromagnet) and the effective dimension of the system becomes $d_{\text {eff }}=d+z$ where $d$ is the geometrical dimension [⿴囗口, 2]. On the contrary, the scaling observed in $\mathrm{CeCu}_{5.9} \mathrm{Au}_{0.1}$ implies that the system is below the critical dimension. The origin of this behavior is believed to be in the anomalous spin dynamics [10] implying that the effective dimension is lower by $1 / 2$ compared to the usual scenario of quantum phase transitions. These results need new theoretical treatement beyond the current understanding of the QCP.

In $\mathrm{Ce}_{1-x} \mathrm{La}_{x} \mathrm{Ru}_{2} \mathrm{Si}_{2}$, a complete softening of $\Gamma_{I S}$ is not observed at the QCP as already discussed. For this system, it is experimentally clear that $T_{N} \rightarrow 0$ at the QCP but that $T_{K}$ stays finite in the ordered phase. This latter quantity may collapse far

$\ddagger$ This point is difficult to be experimentally adressed by INS not only because of the limited access to the lowest temperatures but also because of the instrumental resolution, which will limit the distinction between static and dynamics below a certain energy $\omega$. 
in the magnetic phase. Concerning the temperature variation of $\Gamma_{I S}$, it follows the SF prediction $\left(\Gamma_{I S}=y_{0}+a^{\prime} T^{3 / 2}\right)$ with $a^{\prime} \approx 0.2 \mathrm{meVK}^{-3 / 2}$ as measured for $x=x_{c}$. It is important to note that the value of $a^{\prime}$ is the same for $\mathrm{CeRu}_{2} \mathrm{Si}_{2}$ and thus does not evolve with $P$ or $x$. The same remark holds for the system $\mathrm{CeCu}_{6-x} \mathrm{Au}_{x}$ : The slope of the order unity found de facto in the $\omega / T$ scaling for $\Gamma_{I S}$ is also found for pure $\mathrm{CeCu}_{6}$ [33].

The results obtained on the systems $\mathrm{CeCu}_{6-x} \mathrm{Au}_{x}$ and $\mathrm{Ce}_{1-x} \mathrm{La}_{x} \mathrm{Ru}_{2} \mathrm{Si}_{2}$ are quite different since the former seems to be the paradigm of the strong coupling theory while the latter is the paradigm of SF theory. The behavior observed at a magnetic nonmagnetic QCP is thus not universal. What still remains surprising is that pure $\mathrm{CeCu}_{6}$ and $\mathrm{CeRu}_{2} \mathrm{Si}_{2}$ compounds are very similar while their respective QCP are so different. Regarding the low energy scales involved in $\mathrm{CeCu}_{6}$, it is not excluded that the observed physics corresponds to the fact that the Fermi surface is not yet developped at the temperatures experimentally achievable. To test this possibility, $\omega / T$ scaling must be searched in $\mathrm{Ce}_{1-x} \mathrm{La}_{x} \mathrm{Ru}_{2} \mathrm{Si}_{2}$ for $k_{B} T \gg \Gamma_{S S}$ near the QCP. The idea is that such a scaling may apply in a temperature range above the $T^{3 / 2}$ regime experimentally observed and predicted by SF theory.

\section{Conclusion}

This study is, to our knowledge, the first INS investigation of the QCP performed on single crystal where pressure is the control parameter for the magnetic non-magnetic

phase diagram. The data obtained show a quantitative similarity between the approach of the magnetic phase versus pressure or temperature. Our results are in agreement with the previous experiments performed on alloys near the QCP $\left(x \approx x_{c}\right)$ and in the paramagnetic phase $x<x_{c}$. Our overall data on the compounds of the $\mathrm{Ce}_{1-x} \mathrm{La}_{x} \mathrm{Ru}_{2} \mathrm{Si}_{2}$ family strongly support the SF approach. In the future emphasise will be put on the low temperature data at $P_{c}$ and $x_{c}$ to confirm this picture.

\section{Acknowledgments}

We acknowledge the help of J.M. Mignot for preliminary experiments performed at L.L.B., Saclay. This work benefits from usefull discussions on QCP with C. Pépin, M. Lavagna, P. Haen, K. Ishida, K. Miyake, S. Kambe, B. Fåk and N. Bernhoeft. 


\section{References}

[1] See e.g. Proceedings of the International Conference on Strongly Correlated Electron Systems 2000 Physica B $\mathbf{2 8 1} \& \mathbf{2 8 2}$.

[2] Millis A 1993 Phys. Rev. B 487183.

[3] Moriya T and Takimoto T 1995 J. Phys. Soc. Japan 64960.

[4] S. Sachdev, Quantum phase transitions, Cambridge university press 1999.

[5] Doniach S 1977 Physica B 91231.

[6] Löhneysen H v 2000 J. Magn. Magn. Mat. 200532.

[7] Raymond S et al. 1999 Physica B 259-261 48.

[8] Tabata S et al. 1999 Physica B 259-261 259-261 70.

[9] Stockert O et al. 1998 Phys. Rev. Lett. 805627.

[10] Schröder A et al. 1998 Phys. Rev. Lett. 805623.

[11] Raymond S et al. 1997 J. Low Temp. Phys. 109205.

[12] Kambe S et al. 1996 J. Phys. Soc. Japan 653294.

[13] Lejay P et al. 1993, J. Cryst. Growth 130238.

[14] Quézel S et al. 1988 J. Magn. Magn. Mat. 76 \& 77403.

[15] Kawarasaki S et al., 2000 J. Phys. Soc. Jpn. 69 Suppl. A 53.

[16] Kawarasaki S et al., 2000 Phys. Rev. B 614167.

[17] Haen P et al. 1996 J. Phys. Soc. Japan Suppl. B 6527.

[18] Regnault L P et al. 1988 Phys. Rev. B 384481.

[19] Jaccoud J L et al. 1989 Physica B 156 \& 157818.

[20] Rossat-Mignod J et al. 1988 J. Magn. Magn. Mat. 76 \& 77376.

[21] Kuramoto Y and Kitaoka Y, Dynamics of heavy electrons, Clarendon press, Oxford 2000, 81-83.

[22] Kuramoto Y, 1987 Solid State Comm. 63467.

[23] Haen P et al. 1987 J Low Temp Phys 67391.

[24] Jacoud J L, PhD-thesis, University of Grenoble, 1991.

[25] See e.g. Ziman T and Lindgård P A 1986 Phys. Rev. B 331976.

[26] Broholm C et al. 1991 Phys. Rev. B 4312809.

[27] Raymond S, in preparation.

[28] Mignot J M et al, 1991 Physica B 171357.

[29] Miyako Y et al., 1997 Physica B 230-232 1011.

[30] Pépin C and Lavagna M 1999 Phys. Rev. B 592591.

[31] Lavagna M and Pépin C 2000 Phys. Rev. B 626450 and erratum 2001 Phys. Rev. B 63029901.

[32] Coleman P 1999 Physica B 259-261 353.

[33] Stockert O, private communication.

[34] Hatatani M et al., 1998 J. Phys. Soc. Japan 674002. 